\title{
Tendencias clave para el tercer gobierno del MAS, Bolivia 2015-2019
}

Xavier Albó

Centro de Investigación y Promoción del Campesinado (CIPCA)

\begin{abstract}
This presentation takes into account the 2006 Plan for National Development and aims at highlighting some of the successes in the presidency of Evo Morales as well as the challenges for his third period of government (2015-2019). It begins by reviewing basic demographic information, according to the 2012 Census, and then focuses on the economy: hydrocarbons, mining, agricultural production and the issue of ecology. The quinoa boom, the expansion of the agricultural frontier and the suspension of the construction of a highway through TIPNIS are some of the topics reviewed. Internationally, there are reasons for optimism in relation to Bolivia's demand for access to the sea, while nationally, MAS secured a congressional victory in the 2014 elections. Reforms in the judiciary are the least successful. The presentation ends with an update on social movements that have allowed the formation of the Constituent Assembly, and on the question of municipal, departmental and indigenous autonomies.
\end{abstract}

\section{Keywords}

2006 Plan for National Development, municipal, departmental and indigenous autonomies, quinoa boom, social movements

\footnotetext{
${ }^{1}$ Este trabajo fue presentado en el Primer simposio de bolivianistas organizado por la Sección LASA-Bolivia en la Universidad de Pittsburgh, del 26-27 de marzo 2015. El tema del simposio fue "Des-descolonización, indianidad y despatriarcalización: un debate actual en Bolivia".
} 


\section{Resumen}

Esta presentación tiene como trasfondo el Plan Nacional de Desarrollo y pretende resaltar algunos de los logros del presidente Evo Morales y los desafíos que enfrenta en su tercer gobierno (2015-2019). Comienza revisando información demográfica básica, según el Censo de 2012, para luego centrarse en la economía: los hidrocarburos, la minería, la producción agropecuaria y la ecología. El boom de la quinua, la expansión de la frontera agrícola y la suspensión de la construcción de una carretera que atraviese el TIPNIS son algunos de los temas que se discuten. Internacionalmente, hay motivos de optimismo con relación a la demanda marítima de Bolivia, mientras que, a nivel nacional, el MAS aseguró una victoria parlamentaria en el año 2014. Las reformas menos exitosas son las del órgano judicial. La presentación termina revisando los movimientos sociales que permitieron la formación la de Asamblea Constituyente y el asunto de la autonomía municipal, departamental e indígena.

\section{Palabras claves}

autonomías municipales departamentales e indígenas, boom de la quinua, movimientos sociales, Plan Nacional de Desarrollo 2006

Como trasfondo general de la presentación está el Plan Nacional de Desarrollo: Bolivia digna, soberana, productiva y democrática para Vivir Bien que se concluyó en 2006, a mediados del primer año de la primera gestión del presidente Evo Morales, cuando se estaba poniendo en marcha la Asamblea Constituyente que desembocó finalmente en la actual Constitución del Estado Plurinacional, aprobada y promulgada a principios del año 2009. El Plan fue elaborado en seis meses por el Ministerio de Planificación del Desarrollo, entonces dirigido por el recién fallecido Carlos Villegas, que después encabezó la actual transformación de YPFB, fuente principal de los recursos para ejecutar dicho Plan.

En la página 21 de este Plan se proponía una serie de metas que se deberían haber logrado al concluir su ejecución hacia fines de 2011 y unos rasgos generales que Bolivia debería haber consolidado hasta el año 2015.

Por los grandes giros de la historia, no tiene sentido un cotejo detallado de lo entonces previsto y lo hasta ahora realizado. Pero, en síntesis, lo que mejor se cumple es el nuevo rol internacional de Bolivia y la incorporación de políticas y enfoques de equidad cultural, de género y social, dentro del vivir bien; lo peor, lo referente al órgano judicial. Con una nueva CPE y leyes que 
nos han de permitir generar y redistribuir mayores ingresos, y con una buena gestión, nuestra pobreza bajó al 44,7\% (Censo 2012), incluso por debajo del $49,7 \%$ previsto por el mencionado Plan. Sin embargo, aún no hemos podido modificar nuestra matriz productiva. Ojalá en este quinquenio podamos abocarnos a fondo a la tarea de ampliar y diversificar la base productiva. Debemos institucionalizar todos nuestros procedimientos democráticos dentro del pluralismo previsto por nuestra CPE.

Esta presentación no ha sido pensada a partir de aquel Plan sino para subrayar algunos de los logros y desafíos cualitativos del tercer gobierno de Evo y el MAS (2015-2019). En su exposición, la presentación será temática y cualitativa, sin abrumarles con muchas cifras ni pesadas citas bibliográficas.

\section{Demografía básica}

Según el Censo 2012, Bolivia tenía entonces por lo menos 10,06 millones de habitantes, equivalente a unos 10 por $\mathrm{km}^{2}$. De ellos, un $67,5 \%$ era "urbano" que, en Bolivia, quiere decir agrupado en núcleos de 2000 o más pobladores. Las tres mayores aglomeraciones metropolitanas están en Santa Cruz de la Sierra, La Paz/El Alto y Cochabamba. La propia ciudad de Santa Cruz tiene 1,45 millones y con otros municipios colindantes conforma una región metropolitana de 1,8 millones. La Paz (768 mil) y El Alto (848 mil, la segunda ciudad del país) eran una única ciudad hasta hace dos décadas e incluso hoy es imposible pensar la una sin la otra; y la mancha urbana ya se extiende a otros municipios colindantes, con una región metropolitana también de 1,8 millones. La tercera región es Cochabamba, con 632 mil en la capital, pero la región metropolitana, con otros varios municipios, ya suma 1,2 millones. Es decir, en esas tres áreas metropolitanas se concentra casi la mitad de la población. Las otras seis ciudades más pobladas son: Oruro (265 mil), Sucre (261 mil), Tarija (205 mil) Potosí (191 mil) y Trinidad (102 mil); con ellas ya tenemos la mayor parte de la población urbana del país.

A nivel nacional, según este censo, se estima que el $45 \%$ de la población seguía siendo pobre y solo un $25 \%$ tenía satisfechas sus necesidades básicas. El analfabetismo ya no era un problema grave por cuanto afectaba a un $5 \%$, sin oscilaciones fuertes ni siquiera entre los pueblos indígenas. Y el promedio de años de escolaridad hasta los 19 o más años es de 9,5, teniendo los varones aproximadamente un año más que las mujeres. La principal categoría laboral sigue siendo el trabajo por cuenta propia (50,6\%) que, en algunos pueblos indígenas, puede alcanzar niveles bastante más altos. El sector económico más generalizado (con un 59\%) es el terciario o de servicios. 
El Censo 2012 mostró una paradoja con relación al porcentaje de la población "indígena originario campesina" (IOC, en los términos de la actual Constitución Política del Estado 2009). En el Censo 2001, la población mayor de 15 años autoidentificada como perteneciente a alguno de los 36 pueblos IOC sumaba $62 \%$, siendo los más numerosos, por mucho, los quechuas (32\%) y luego los aymaras (25\%). En cambio, en el Censo 2012, en el sexto año de la presidencia del aymara Evo Morales, la cifra bajó sorpresivamente al $41 \%$, lo que dio lugar a comentarios de toda índole.

Aclaradas las cosas, ahora ya me resulta patente que un factor clave de este descenso fue la nueva y compleja formulación de la siguiente pregunta: ${ }^{2}$ "Como boliviano o boliviana, ¿pertenece a alguna nación o pueblo indígena, originario, campesino o afroboliviano? Respuestas NO / sí ¿a cuál?”. Respuesta libre con apoyo de una lista de casi 40 grupos étnicos que, salvo alguno, ya habían sido identificados en el censo anterior. La primera respuesta filtro (Sí/NO) y la compleja formulación de la pregunta, al parecer confundió más que ayudar y muchos, sobre todo ya citadinos, para no complicarse la vida, respondieron "NO". Los dos grupos principales siguen siendo por mucho los quechuas y aymaras. ${ }^{3}$ A consecuencia de ese cambio ya no tiene sentido comparar en este punto los datos del 2001 y 2012. Una útil innovación de este censo es que por primera vez se contó a la población afroboliviana, arrojando una cifra total de apenas $23.330(0,2 \%)$, mucho más baja que en otros países del continente. En un $70 \%$ ya viven en ciudades y su nivel de pobreza es prácticamente igual que el promedio nacional: $44,7 \%{ }^{4}$

2 Meses antes, el mismo 2012, se realizó una nueva ronda de la encuesta LAPOP, sobre la democracia en el país con un sofisticado marco estadístico. En ella se mantuvo la formulación del Censo 2001 y, con ella, el porcentaje de IOC subió más bien a 71\%. Dime cómo preguntas y te diré cuántos somos.

$317,3 \%$ quechuas y $15,1 \%$ aymaras, más un número impreciso (no muy elevado) que, siendo de estos dos orígenes y lenguas, se autoidentificó con otros nombres más locales. En las clasificaciones ulteriores de esa pregunta abierta, el INE ha elaborado tentativamente tres categorías: a) Naciones y Pueblos (NyP) IOC mayoritarios (aymara y quechua); b) NyP IOC minoritarios; y c) otras denominaciones. Esta última es aún un cajón de sastre, aunque por coherencia estadística ya se eliminaron muchas respuestas con 100 o menos casos. Es un primer paso que necesita pulirse.

${ }^{4}$ Los leko, aguas abajo de Yungas hacia Apolo, son un caso singular de etnogénesis: se enumeraron sólo 9 en el censo rural indígena de 1994 (seguramente por considerar sobre todo su idioma); ya se estimaban 4.186 en el Censo 2001 y en 2012 se han autoidentificado 13.527 con un alto índice rural (75\%) y masculinidad (115\%); en su mayoría hablan quechua o solo castellano. La causa probable es querer consolidar su TCO/TIOC (Tierra Comunitaria de Origen / Territorio Indígena Originario Campesino) 


\section{Rasgos económicos}

\section{Hidrocarburos y el shale gas}

Parece que el descenso del precio de los hidrocarburos, de precios superiores a los cien dólares por barril a 50/barril, e incluso menos, ya es algo inevitable. Se debe en buena parte a forcejeos, por parte de los productores tradicionales de la OPEP, para impedir que los menores costos logrados sobre todo por Estados Unidos con su nueva tecnología de fracking y shale gas o de esquisto se difunda a sus países. La "pulseta" todavía tiene oscilaciones pero no parece que lleguemos a los niveles anteriores.

\begin{tabular}{|l|c|c|c|}
\hline \multicolumn{5}{|c|}{ Figura 1 - Reservas de gas natural y potencial de Shale gas } \\
en América Latina
\end{tabular}

En shale gas Bolivia es el quinto país en posibilidades, muy por debajo de los primeros países, a menos que mayor inversión en investigación nos demuestre lo contrario. En este nuevo recurso Venezuela tiene también muchas menos posibilidades, comparada por ejemplo con México, Argentina y Brasil-estos dos últimos son actualmente los principales compradores de nuestro gas.

Esta tecnología, ya muy difundida en los Estados Unidos, sigue siendo controversial y poco clara desde la perspectiva medioambiental, sobre todo por las dos razones siguientes: la contaminación de áreas del subsuelo (por no poder controlar todas las inyecciones de diversos productos químicos) y la necesidad de grandes recursos de agua. Habrá que estar muy al tanto de cómo evoluciona todo ello en los próximos años. 


\section{Minería}

Desde las relocalizaciones de los años 1980, con motivo de los precios bajos de la minería, los costos elevados (en parte por la cantidad de mano de obra) y la reducción de la Corporación Minera de Bolivia (COMIBOL) a una empresa "residual", las cooperativas mineras fueron sustituyendo a los viejos sindicatos, salvo en algunas empresas en especial rentables como Huanuni, Bolívar (en su momento) y Colquiri.

Ya en el año 2006 uno de los principales conflictos laborales que debió enfrentar el flamante presidente Evo Morales fue el de cooperativistas y sindicalistas de COMIBOL en la mina de Huanuni, donde ambos sectores ambicionaban el control del yacimiento más rentable de Posoqoni. Sobre todo por explosiones de cartuchos de dinamita, hubo 16 muertos y decenas de heridos. En este caso la solución pasó en parte por la reincorporación masiva de bastantes cooperativistas a la planilla de sueldos de COMIBOL. La ventaja de ello es que tienen mayor seguridad laboral, aunque pierden las ventajas ocasionales de ganancias más rápidas en una buena veta y con picos de buenos precios. Aquel incidente ratificó que también entre proletarios mineros puede haber pugnas parecidas a las del "capitalismo salvaje" del Far West. A mí siempre me ha llamado la atención la facilidad con que entre los mineros andinos puede mantenerse un diálogo permanente entre Marx, con su crítica al capitalismo, y el Tío al interior de la mina, como expresión de la más honda cosmovisión ancestral. Algo semejante ocurrió en Colquiri en el año 2013, en este caso sobre cómo repartir los niveles superiores e inferiores de una misma veta. La solución fue semejante y, por suerte, solamente hubo un muerto en una marcha que ambos sectores hicieron hasta La Paz. ${ }^{5}$

Hay actualmente tres tipos de manejo laboral en la minería:

1. Los antiguos sindicatos y algunos nuevos sindicatos de algunos yacimientos especialmente buenos.

2. Las "cooperativas", que son la gran mayoría, gozan además de un marcado soporte por parte del gobierno debido a su decidido apoyo al actual

\footnotetext{
${ }^{5}$ Esta vez al finado, que estaba en el techo de la sede de la Federación de Mineros, le había impactado un cartucho de dinamita lanzado con honda por los cooperativistas. A mí me tocó celebrar la misa por él en pleno paseo del Prado, junto a la antigua sede de la COB, exactamente en el mismo lugar donde el primero de mayo de 1978 se concentró la marcha de la $\mathrm{COB}$, la primera que podía realizarse de forma masiva después de la exitosa huelga de las cuatro mujeres mineras y sus 15 hijos con el apoyo de más de mil huelguistas por todo el país. Fue el principio de la caída del dictador militar Hugo Banzer. Tremendo contraste.
} 
proceso desde un principio. Pero en ellas se debe distinguir también entre los "dueños" de la cooperativa que, a veces, se van pareciendo a los empresarios privados o estos últimos quieren incluso hacer convenios de joint venture con ellos para evadir impuestos (que, por cierto, siguen bastante bajos en el sector de la minería), y los trabajadores asalariados precarios y muy oscilantes, al servicio de ambos sin poder entrar en la cooperativa, al menos de momento, hasta ganarse su derecho de piso.

Un caso particular es el de nuevos yacimientos de oro que aparecen y desaparecen según los precios coyunturales, y que provocan periódicamente conflictos comparables a los arriba mencionados. En teoría son trabajados también por cooperativas, muchas de ellas sin papeles legales, aunque en este caso pueden entrar en conflicto también con los comunarios locales que tienen sus títulos ejecutoriales, a veces colectivos. Ejemplos: Arcopongo en Sud Yungas e Inquisivi; Apolobamba tocando la frontera con Perú; Malku Quta, en el norte de Potosí, donde existe un componente curiosamente llamado "indio" en la tabla de los 94 elementos y que, de podérselo aislar con sofisticada tecnología, podría añadirle un importante valor agregado.

3. Varias empresas privadas sofisticadas y vinculadas a multi-nacionales, que usan nuevas tecnologías a tajo abierto con mucho capital y pocos trabajadores. Estas últimas son las que más recursos exportan al exterior. El caso más notable es el de la Empresa Minera San Cristóbal en Sud Lípez que ya ha pasado por varias manos hasta su administración actual, japonesa. Inició su trabajo en 1996 y empezó a exportar plata, zinc y, en menor grado, también plomo a partir del año 2006. Con su producción, la estructura de exportación minera ha cambiado significativamente: las principales exportaciones son ahora la plata y el zinc, mientras el histórico estaño ha quedado relegado al cuarto lugar.

Este tercer modelo se parece al del boom minero del Perú y Chile pero en menor escala, porque en Bolivia sigue pesando mucho la mano de obra local y la relación con las organizaciones de trabajadores, sean sindicatos, cooperativas o comunidades. En contraste con la Empresa San Cristóbal que, en conjunto solamente emplea a 500 personas, en la Cooperativa Unificada de Potosí (del modelo 2) hay 5000 trabajadores, de los que un 60\% están afiliados a la cooperativa y los demás son asalariados eventuales.

La metáfora de la película Avatar ha sido ya usada por los esposos Anthony y Denise H. Bebbington quienes, al estudiar el fenómeno minero contemporáneo en los países andinos, muestran la necesidad de lograr algunas formas de glocalización que combinen los intereses locales desde 
abajo y los globales desde arriba tanto en la minería como en otros recursos naturales no renovables.

\section{Producción agropecuaria y ecología}

Son notables las mejoras en muchas áreas del campo en infraestructura para servicios básicos como caminos, electricidad, edificios escolares, postas de salud y otras obras de dotación básica. Se va continuando así lo que ya se había iniciado en 1994 con la llamada Ley de Participación Popular (ver infra), pero con bastantes más recursos. Uno de los ámbitos más difíciles y necesarios es el del riego, para mejorar la producción rural propiamente dicha

El principal producto altiplánico que ha logrado disparar sus precios en el mercado internacional en los últimos años es, sin lugar a dudas, la quinua real, sobre todo en el contorno del salar de Uyuni. Ello tiene sus efectos muy favorables con relación a la economía de estas regiones antes bastante deprimidas. Pero hay que tener también cautela:

- Cara a la comercialización, hay que cuidar la buena calidad del producto para mantener esos buenos precios. El año 2014 hubo problemas, atribuidos a que se mezcló quinua local de buena calidad con otra peruana no tan buena. Además, con esos precios, es más probable que otros países que nunca habían producido quinua logren adaptarla a sus climas no tan extremos y quizás obliguen a bajar los precios. Habrá que estudiar bien las diversas especies de quinua, sus particularidades y los nichos en que podemos mantener ventajas comparativas.

- En segundo lugar, cara a la gestión del suelo, con el boom de la quinua se va modificando también la estructura productiva de muchas comunidades. Un caso típico son las zonas de bofedales, fundamentales para las actividades pastoriles con los camélidos. Si se trasfieren al cultivo de momento más lucrativo de la quinua, podría crearse un desequilibrio a la larga. Cerca del lago Poopó ha habido problemas en comunidades con títulos colectivos, porque algunos miembros que ya no iban hasta allá por dedicarse a otras actividades no agrícolas más lucrativas han retornado, pero con cierta prepotencia, cercando con alambradas terrenos que son legalmente para todos o incluso de terceros, los uru muratos.

Hay que seguir manteniendo la sabiduría andina, y de tantos otros productores, que es la de no poner todos los huevos en una única canasta y la sabiduría de la convivencia entre los distintos. 
En el ámbito rural oriental, señalaré dos pistas:

- La primera son las innovaciones que se están desarrollando en varias partes de la Amazonía hacia un modelo alternativo de sistemas agroforestales que buscan una mejor simbiosis entre el monte y sus productos, por un lado, y otros cultivos complementarios, por el otro, para aumentar la capacidad comercial y productiva de la selva sin transformar sus bosques en pastizales.

- La segunda pista de análisis es el tira y afloja actual entre el apoyo del Gobierno (1) a los pequeños productores y colonizadores (hoy llamados interculturales) y a los indígenas, todos ellos aliados iniciales del presente gobierno, y (2) últimamente también a los productores medianos y grandes. Volveré enseguida al tema.

En lo ecológico, más allá de lindas retóricas -como la Ley Corta sobre la "Madre Tierra" (MT), la parte teórica de la posterior Ley Marco sobre la MT y nuestros bellos discursos a nivel internacional-, estamos aún bastante mal. Es tremenda la basura ecológica que dejan muchas minas y la basura de todo tipo que generan las periferias urbanas. Oruro es quizás el ejemplo más grave.

Se ha montado ya una oficina nacional en Defensa de la Madre Tierra, pero la última vez que la visité, poco antes del nuevo período gubernamental, estaba constituida casi solo por varones y collas, con unos recursos humanos y monetarios mucho menores, por ejemplo, que los de la oficina del gobierno departamental de Santa Cruz que se dedica mucho más a la expansión de la frontera agrícola. En efecto, en los últimos años y, pese a estar en pleno año para la economía rural familiar, el Gobierno ha estado dando prioridad a los productores mayores del Oriente (que apenas son el 6\% del total), para la seguridad alimentaria. Se ha señalado la meta de ir ampliando la frontera agrícola de unos tres a unos trece millones de hectáreas, a costa de áreas todavía boscosas. Pero no es fácil el equilibrio entre ambos sectores. ¿No se beneficiarán sobre todo esos grandes productores, mientras los pequeños productores familiares seguirán siendo los marginados de siempre?

Veremos si y por cuánto tiempo resistimos a los transgénicos, incluso de productos muy nuestros, como la quinua, con su momentáneo boom internacional al que ya nos hemos referido. Esto es parte también de la pulseta con la quinua peruana.

Otro tema es el de la relación dialéctica entre la hoja de coca con sus usos tradicionales, que en nuestro caso son incluso parte de nuestra actual CPE, y la política de control de las drogas. Ya hace años que se inició un estudio auspiciado por la Unión Europea sobre cuánta superficie con cultivos de hoja de coca se necesita para sus usos tradicionales. Se tardó tres años en 
difundir sus resultados y al final se adoptó una solución salomónica, fijando la necesidad en 14.7 mil hectáreas, con lo que se amplió algo la cantidad inicialmente recomendada por el estudio para tomar en cuenta otras actividades tradicionales allí posiblemente no consideradas. La existencia de ADEPCOCA (Asociación de Productores de Coca de los Yungas de La Paz), que permite cubrir todo el circuito del productor a los consumidores, según cupos y vínculos preestablecidos, ha dado cierta estabilidad al precio de la hoja de coca, por lo menos a la yungueña. Se consideró un triunfo la aceptación del akulliku en Viena, siquiera para Bolivia y sin que ello marginara al país de convenios internacionales.

Hay muchos más interrogantes sobre la coca del Chapare de la que solo en torno a un $8 \%$ pasa por el centro de acopio y control de Sacaba, una especie de ADEPCOCA en Cochabamba. Veremos en qué quedan las disputas sobre cuántas hectáreas de esa coca legal extra, acordada tras la presentación del estudio, corresponden a Yungas y cuántas al Chapare. Algo constatable en casi todas las áreas productoras de coca es que la población local ha perdido interés en producir allí otras frutas y productos como cítricos, plátanos o café, porque la coca da mayores beneficios. Cabe resaltar también que es poca la coca/cocaína boliviana que va a parar a los mercados norteamericanos.

El tema de la carretera por el TIPNIS que tanto tiempo, energías y dinero consumió entre 2011 y 2013, quedó congelado en vísperas de las elecciones de fines del 2014. La Ley 180, que frenó esta carretera, había arrancado por la VIII marcha indígena de fines del 2012 al haberse fortalecido con un recibimiento triunfal en La Paz después del fiasco en que se metió el Gobierno en el fracasado intento de dispersarla en Chaparina. Evo se vio acorralado y tuvo que aceptar esa ley a desgano.

Pero casi inmediatamente Evo preparó una anti-marcha liderada por CONISUR (Consejo Indígena del Sur) ${ }^{6}$ con indígenas de dentro y fuera del TIPNIS que sí quieren la carretera, porque tienen muchos vínculos con los cocaleros asentados en esta parte (aunque se evitó que participaran en la marcha). Fueron por la ruta de Cochabamba, llegaron a La Paz sin contratiempos, allí fueron enseguida recibidos por el Presidente, y en pocos días el Parlamento aprobó la Ley 222 que solicitaba muy tardíamente la consulta "previa" sobre la carretera.

${ }^{6}$ Escisión surgida en 1997 de la antes única subcentral TIPNIS en la región sur del TIPNIS, que la prefectura de Cochabamba reclama como suya en la frontera departamental hasta ahora no definida. 
Con gran despliegue de recursos y reiteradas visitas a las comunidades más reticentes, se realizó esa consulta que, en los hechos, se centró en listar primero comunidad por comunidad sus prioridades de desarrollo y enseguida presentar a cada comunidad una disyuntiva: “¿Qué prefieren? ¿Ese desarrollo o la intangibilidad absoluta del Parque?". La consulta se apoyó con un doble mapa del TIPNIS: uno totalmente rojo, indicando que no podía tocarse nada y el otro en el que se distinguían internamente tres regiones dentro del Parque, según sus niveles de intangibilidad, definidas ya desde antes por el SERNAP (Servicio Nacional de Parques). Todas menos la primera comunidad consultada dijeron como era natural "el desarrollo", aunque una docena de comunidades se negó hasta el final a participar en la consulta. Los mismos que habían realizado la VIII Marcha iniciaron otra IX Marcha que llegó también sin contratiempos a La Paz, pero nunca fue recibida por el Gobierno y al final tuvo que retirarse sin pena ni gloria. Se demandó asimismo la inconstitucionalidad de la Ley 222 y el Tribunal Constitucional respondió de una forma medio sibilítica que esa Ley es constitucional siempre que se concierten previamente el protocolo y sus mecanismos con las organizaciones indígenas.

Esta concertación nunca se realizó de una manera general, pero la consulta siguió su curso explicando el procedimiento a los participantes al llegar a cada comunidad. Los líderes de la VIII y la IX Marcha recurrieron a la Iglesia boliviana y, a través de la Asamblea Permanente de DDHH en Bolivia, a la Comisión Interamericana y a la Federación Internacional de DDHH, a fin de verificar la calidad de aquella consulta "previa", pero tan tardía. En sus conclusiones dijeron que esa consulta no cumplió los estándares esperados, motivo por el cual entretanto no podía construirse la carretera. Ya se estaban acercando las elecciones de fines del 2014 y el Gobierno decidió priorizar las necesidades de desarrollo en la región congelando el asunto de la carretera. Esta decisión de momento se mantiene. En su discurso de inicio de su tercer período, el 22 de enero 2015, Evo Morales criticó aquellas movilizaciones que obligaron al Gobierno a devolver fondos al Brasil, pero solo habló de otras dos carreteras entre Cochabamba y Beni que no tocan el Parque. Nunca llegó a derogarse tampoco la Ley 180.

\section{Estabilidad financiera y diversificación econó- mica}

Detrás de estos y otros muchos emprendimientos del país, llama la atención la gran estabilidad financiera de Bolivia, sin grandes sobresaltos. 
Pese a la retórica y práctica "nacionalizadora" del país sobre diversos recursos naturales y a haber dejado de recibir los créditos condicionados del Fondo Monetario Internacional, el país ha merecido la alabanza de diversos entes financieros internacionales por su estabilidad macroeconómica y su capacidad de tener ahora un buen colchón financiero frente a los nuevos bajones de precios. Seguramente podremos resistir todavía uno o dos años. Hay que reconocer al Ministro de Economía, Luis Arce Catacora, que hasta ahora ha sabido torear en el país las diversas crisis del contorno cercano o distante. Son, junto al canciller David Choquehuanca (con un perfil totalmente contrapuesto) y Juan Ramón Quintana (que solo ha cambiado de ministerio), los tres ministros que siguen estables en el Gabinete desde 2006.

Hasta ahora gran parte de los cambios ocurridos en el país han sido posibles gracias a los mayores recursos generados sobre todo por la ley de "nacionalización" del 2006. Y con ellos se han ido generando o mejorando diversos bonos para que los beneficios lleguen directamente a la población $y$, con ello, aumente también el circulante: tanto los mecanismos de la participación popular, base de todo el fortalecimiento del nivel municipal, como el Bonosol, ambos iniciados por Gonzalo ("Goni") Sánchez de Lozada, tienen ahora muchos más recursos; el SUMI para el binomio madre-hijo; el bono Juancito Pinto para los alumnos, etc. En eso Bolivia está replicando modelos que también han tenido mucho éxito en Brasil, aunque no llegan aún a modificar la estructura productiva, propiamente dicha.

Ahora parece acabarse el período de las "vacas gordas" y el Gobierno tendrá sobre sí la gran tarea de diversificar más las fuentes de ingresos, hacia una "economía de base ancha". Evo ya advirtió que llegó el momento de ajustarnos los cinturones.

Hay varias iniciativas hacia la diversificación en una serie de ámbitos, con distintos niveles de éxito. Por ejemplo, las múltiples iniciativas de "compro boliviano", empezando por los desayunos y almuerzos escolares; la fábrica de computadoras quipus para llevar tecnología a todas las escuelas y colegios del país; la casi lista planta de licuefacción y diversificación de gases según su diverso valor comercial, en Villamontes, y la planta de transformación de hidrocarburos en urea en Bulo Bulo; la todavía experimental fábrica de baterías de litio para acumular energía...

A la vez, a Evo Morales le gustan mucho los megaproyectos, como para poder decir que Bolivia es cada vez menos dependiente de los países más desarrollados. Así, lanza el satélite Tupac Katari (todavía con bemoles en su cobertura y en su funcionamiento); construye un gran edificio para el 
funcionamiento del Gobierno central y un sinfín de casas de gobierno, escuelas, coliseos, canchas con pasto artificial, a lo largo y ancho del país; los teleféricos; grandes inversiones para Santa Cruz con ocasión del encuentro del Grupo de los 77 y China; su propio y suntuoso vestuario actual, tan distinto de aquella chompa a rayas con que empezó... La misma carretera por el TIPNIS, como un modelo de carretera ecológica con sus túneles y puentes en medio de la selva, pretendía ser otro megaproyecto. Ha anunciado ya un programa para desarrollar energía nuclear en Bolivia, cuando ni siquiera tiene materia prima para ello, y precisamente cuando otros países del primer mundo -como Japón y Alemania- ya están desmantelando ese tipo de proyectos para sustituirlos con otros de energía renovable, como la solar y la eólica... En todo eso Evo es muy distinto de su amigo Pepe Mujica, el carismático y sencillo ex-presidente izquierdista del Uruguay, enamorado del cultivo de la tierra, que sigue vistiendo sencillamente y manejando su vieja peta.

De todos esos, el megaproyecto más discutible es seguramente el de la energía nuclear y el más funcional y exitoso es quizás el de los teleféricos de La Paz, esa ciudad de grandes pendientes y desniveles como ninguna, que ya ha sido seleccionada como una de las siete ciudades maravillas del mundo. Nuestros tres primeros teleféricos para uso masivo transportan a diario a miles de pasajeros mucho más rápidamente que los micros y a veces hasta que los taxis, por precios muy competitivos. Se espera que en dos años haya otras seis líneas. Aparte de su belleza, silencio y comodidad, el teleférico está modificando poco a poco la relación y entendimiento entre El Alto y La Paz, los polos centrales de esta polarizada conurbación metropolitana. En sus cientos de cabinas se encuentran diariamente gente de las dos urbes que, además de ganar tiempo, tienen ahí preciosos minutos de relax, gozan del espectáculo, sacan fotos y entablan conversaciones entre ellos; miran y se ríen al ver las "trancaderas" del otro transporte pocos metros más abajo. Se va creando también una nueva cultura de respeto y convivencia. Son ahora muchos más los alteños que los fines de semana pueden bajar y pasear, por ejemplo, por el Megacenter, que hasta hace poco era muy exclusivo. Algunos se han quejado, pero prevalece el mayor y mejor intercambio; incluso ha habido ya apthapis (comidas conjuntas en el suelo). Ojalá todo ello sean los primeros pasos para un mayor entendimiento intercultural entre unos y otros, más allá de las diferencias étnicas y políticas, y también, mediante un buen sistema de transbordos y tarjetas para poder intercambiar medios de transporte, un paso más hacia la transformación de todo el transporte (inter)urbano, todavía muy caótico, pues los teleféricos, aunque 
bastante usados, cubren a lo más el $15 \%$ de ese transporte.

El contrapunto es que algunos proyectos más baratos y cercanos a la gente pero menos espectaculares, por ejemplo en la dotación de hospitales y en la atención a los usuarios, van quedando retrasados. Con frecuencia el cuello de botella es la falta de recursos humanos suficientemente formados y motivados. Al principio del primer mandato de Evo vinieron al país numerosos médicos cubanos que ayudaban de forma gratuita, sobre todo en problemas de la vista, pero chocaron mucho con organizaciones locales de salud. Más adelante han ido a Cuba unos cinco mil becados para medicina que ahora ya están retornando.

\section{Rasgos políticos}

\section{$\mathrm{Al}$ interior de Bolivia}

\section{La victoria del MAS en diciembre 2014}

De momento en la Asamblea Legislativa Plurinacional, el MAS -mediante la matemática electoral del $61 \%$ del voto directo-ya obtuvo los dos tercios en ambas cámaras. El margen se ha ampliado cómodamente poco después con la alianza lograda con diputados del MNR y algún tránsfuga. A ese nivel, por tanto, la nueva gestión del MAS no tendrá mayor conflicto para seguir aprobando "sus" leyes, como en la gestión anterior. Aunque queda por ver si funcionará igual la lealtad partidaria de los "levantamanos" mudos o si los disidentes se animarán a expresar más abiertamente sus diferencias. Hace poco uno, que se quejaba de las imposiciones inconsultas, me consultó: “¿Hasta dónde puedo llegar sin arriesgar mi pega?”.

La principal sorpresa del nuevo escenario político fue quizás la opción por el periodista José "Gringo" González para presidir el Senado y, como contrapunto, la marginación de Carlos Romero, que había estado en ascenso continuado y que en la elección nacional había logrado, por primera vez, el triunfo del MAS en Santa Cruz con un 49\%. Morales ha optado más bien por alguien de reconocida trayectoria popular que, en el pasado, fue su secretario privado, cónsul en la Argentina y embajador en Brasil; y precisamente en un cargo en que pocos años antes había triunfado la hoy disidente Rebeca Delgado... Habrá que estar al tanto de las consecuencias prácticas de esta opción en el cargo gubernamental más importante después de los históricos Evo y Álvaro. Fuera de eso no hay señales de algún cambio de enfoque en el 
nuevo gabinete con los colaboradores inmediatos del Presidente, pese a que casi la mitad han cambiado.

\section{Las elecciones subnacionales del 29 de marzo 2015}

Recién después de las elecciones subnacionales del ya cercano domingo 29 de marzo, tendremos el escenario político completo para el quinquenio. A ese nivel subnacional suele haber siempre diferencias significativas con relación a los resultados de nivel nacional en una misma jurisdicción, por la existencia de otras organizaciones y liderazgos a nivel local. Pero, a un mes de esas elecciones, aún no me animo a hacer predicciones, sobre todo por no poder ponderar suficientemente hasta qué punto seguirá prevaleciendo la figura de "Evo" en los electores, tras el perfil de cada candidato concreto.

El tema más cuestionado fue una controvertida "circular" del Tribunal Electoral Plurinacional que descalificaba como candidatos a alcaldes y otros cargos del mismo nivel a quienes en los dos años anteriores hubieran servido, por ejemplo, en la Asamblea Legislativa fuera de su jurisdicción. Pero, esa prohibición no alcanzaba a los candidatos a gobernadores departamentales, la instancia en que más ha apostado el MAS. La víctima más sonada (pero no la única) fue la disidente Rebeca Delgado, ex presidenta de Senadores por el MAS, que se presentaba como alcaldesa favorita para Cochabamba. En bastantes áreas rurales ha habido quejas por haberse impuesto candidatos elegidos a dedo por el MAS ignorando los propuestos por las bases. ${ }^{7}$

\section{Reformas en el órgano judicial}

En su discurso inaugural, Evo privilegió los tres puntos en los que más debía insistirse en ese nuevo período: la administración de la justicia, la salud y el desarrollo. De ellos el primero es el más estructural, por lo que Evo ya anunció un referendo que podría llevar a un cambio constitucional en este punto. En efecto, la nueva CPE de 2009 ensayó una innovación poco común, al definir que las autoridades del poder judicial debían ser elegidas por el

\footnotetext{
7 El resultado de las elecciones subnacionales (cuyo objetivo fue elegir 9 gobernadores y vicegobernadores departamentales, asambleístas departamentales, 339 alcaldesas o alcaldes de municipio, y 339 concejales para esos municipios) le dieron al MAS una victoria relativa: ganó 6 de las 9 gobernaciones departamentales del país, 8 de las nuevas asambleas legislativas departamentales y 225 municipios; pero perdió el control de importantes ciudades capitales, incluidas la ciudad de La Paz y El Alto, donde el MAS había sido invencible desde 2005.
} 
pueblo y no internamente solo por la Asamblea Constituyente. No es algo totalmente nuevo: Estados Unidos es uno de los pocos países en que ciertos cargos judiciales son también elegidos por la población.

La razón de fondo para el cambio era el descontento general con este órgano del Estado, seguramente el más corrupto y cuestionado. Es también el único que, en virtud de la independencia de cada órgano, rechazó reducir sus sueldos, superiores a los del Presidente.

Sin embargo, la manera concreta con que se eligió a las autoridades judiciales por primera ( $y$ de momento única) vez, no dio los buenos resultados esperados. Muchos piensan que los candidatos fueron nombrados demasiado a dedo por su cercanía al MAS (aunque algunos han resultado más críticos de lo inicialmente pensado, como el juez Gualberto Cusi, representante indígena en el TCP). En realidad, quienes ganaron por mucho la elección fueron los votos blancos y nulos, sea por no conocer a los candidatos o -en el caso de la mayoría de los nulos- por una deliberada acción de protesta. Esto no tuvo mayores consecuencias prácticas, puesto que de todas maneras quedaron posesionados los que obtuvieron mayor votación, aunque sus votos fueran solo el $20 \%$ del total.

En ese órgano del poder judicial hay también otro tema hasta ahora mal resuelto: el del pluralismo jurídico (con la histórica justicia ordinaria, que era antes la única, y las múltiples justicias indígena-originarias, ambas con igual jerarquía constitucional). La relación entre ambas ya fue objeto de enmiendas en la última fase más política, posterior a la Asamblea Constituyente y de ahí surgió después la Ley de Deslinde Jurisdiccional que, de seguro, con un tribunal suficientemente autónomo, no pasaría un test de constitucionalidad, sobre todo por no respetar de manera suficiente esa igualdad jerárquica constitucional. Así lo han comentado autoridades en esa temática de gran prestigio internacional. Un alto dirigente indígena me comentaba que la justicia indígena originaria se había reducido a pleitos de gallinas y otros temas baladíes.

\section{Política internacional}

Evo y el MAS han logrado proyectar la imagen internacional de Bolivia mucho más que cualquier otro gobierno anterior, sobre todo en temas como los derechos de la Madre Tierra, calentamiento global y otros ecológicos. Sigue vendiéndose bien la imagen de Evo como el "presidente indígena" y se 
han promocionado exitosamente coyunturas internacionales como la presidencia temporal del Grupo 70 más China o los contactos directos de Evo con el Papa Francisco, que si no surgen contratiempos de última hora nos visitará en julio de este año. ${ }^{8}$

Pero con frecuencia hay desfases entre lo retórico, sobre todo a nivel internacional, donde hay mayor espacio para elucubrar y teorizar, y los logros reales, sobre todo a niveles más locales donde se prioriza lo concreto y operativo y a lo que ya me referí antes.

Nuestro principal tema internacional es todavía la centenaria demanda marítima ante Chile que ha sido llevada por este gobierno al Tribunal Internacional de La Haya, para la que el Gobierno ha sabido involucrar a gente de primer nivel, como los ex-presidentes Eduardo Rodríguez Veltzé, en La Haya, y Carlos Mesa, como su principal portavoz internacional. La sentencia de La Haya en los límites marítimos entre Chile y Perú, favorable a este último, y el reciente reencuentro entre Evo y Bachelet en Costa Rica alientan un optimismo moderado. ${ }^{9}$

Pero hay también puntos oscuros en la relación con algunos aliados dentro de América Latina. ¿Qué pasará por ejemplo en nuestra relación con los países del Alba si sigue deteriorándose la estabilidad económica y política de Maduro en Venezuela? ¿Y qué pasará con Cristina y Argentina? ¿En qué quedarán nuestras principales exportaciones de gas a este país que es también el más avanzado en la nueva tecnología del shale gas, sobre la que nos referimos al principio? Dilma Rousseff descongeló cierta tirantez que tuvo desde un principio con Evo (en contraste con la cercanía de Lula) al asistir a la toma de posesión de su tercer período; pero también ahí pueden ocurrir sorpresas con el shale gas.

Seguirá la apertura pragmática de Bolivia a todos los países, de cualquier ideología. La reanudación de relaciones entre Cuba y EE.UU facilitará también una mejor relación entre Bolivia y EE.UU que, por otra parte, despliega ahora en esas latitudes menos intervencionismo que en el pasado. Hemos vivido ya varios años sin embajador, USAID ni DEA y no pasa nada grave. Lo más cercano a una crisis sucedió en el año 2013 cuando retornando de Moscú se prohibió el paso de Evo sobre el espacio aéreo de varios países europeos por la sospecha infundada de que en el vuelo se ocultaba a Snowden, el célebre

\footnotetext{
${ }^{8}$ La visita del Papa Francisco a Bolivia se concretó el 8 de julio de 2015.

${ }^{9}$ El 24 de septiembre de 2015 el Tribunal Internacional de La Haya aceptó la demanda marítima de Bolivia.
} 
filtrador de información confidencial. Este episodio creó más bien una nueva aureola de celebridad internacional para Evo.

\section{El MAS y los movimientos sociales}

Desde su surgimiento, este gobierno se ha caracterizado por haber nacido de los movimientos sociales, y más específicamente de las cinco organizaciones indígena originario campesinas que firmaron su "Pacto de unidad" para la Asamblea Constituyente. Fruto de este pacto es la larga frase concertada que más se repite en la actual CPE: "las Naciones y Pueblos Indígena Originario Campesinos", como la manera de referirse a sí mismos y ser reconocidos, con sus diversos nombres locales según el lugar y la organización, como miembros de pueblos preexistentes desde antes de la República y hasta de la Colonia. Gracias a este Pacto avanzó la CPE. Pero el conflicto del TIPNIS ya mencionado lo rompió en dos grupos conocidos como las "trillizas" y las "mellizas". Las primeras son las tres organizaciones más antiguas: 1) La CSUTCB (Confederación Sindical Única de Trabajadores Campesinos de Bolivia); 2) las "Bartolinas", que al principio era la rama femenina de la CSUTCB y después se autonomizó; 3) los "colonizadores" ahora llamados "interculturales".

Estos fueron los grupos que crearon al MAS como su "instrumento político" y, por tanto, lo consideran como su "hijo", al que no pueden abandonar. Las "mellizas" (CIDOB y CONAMAQ) en cambio se crearon aparte y solo se aliaron al MAS con ocasión de la CPE; pero cuando estalló el conflicto del TIPNIS rompieron definitivamente con el MAS y se perdió aquella unidad que había sido instrumental para la CPE.

EI MAS optó por reconstruir el Pacto pero por la vía de dividir a las "mellizas", promocionando en ambas una rama más dócil a este partido. Empezó a hacerlo con la Confederación de Pueblos Indígenas de Boliva (CIDOB), creada en 1982, con la que ya había tenido sus conflictos en el TIPNIS; por tanto hay ahora el CIDOB histórico, que sigue dirigiendo el takana Adolfo Chávez, protagonista en las marchas del TIPNIS y antes en la Asamblea Constituyente, y otro CIDOB-Masista dirigido por la moré Melva Hurtado, que ahora vive en Trinidad.

EI CONAMAQ, más centrado en la región andina, se había consolidado como organización nacional recién a fines de 1990, aunque desde antes tenía antecedentes a niveles más locales. No habían faltado algunas divisiones 
internas en el pasado entre sectores más gubernamentales, antes del ascenso del MAS, y otros más autónomos. Cuando recién estaba naciendo, había hecho grandes esfuerzos en distinguirse de la CSUTCB por tener un discurso más explícitamente étnico, sobre todo en regiones de ayllus o incluso reconstruyéndolos en lugares donde ya se habían perdido. En toda esa tarea había recibido ayuda de la ONG danesa IBIS, entre otras, y esa fue una de las causas que precipitó la expulsión de esa importante financiadora danesa, sin ningún delito formal, en 2013. Al perder ese importante apoyo, el CONAMAQ histórico u "orgánico" disminuyó su presencia y entonces el MAS remató su estrategia creando su rama más leal, dirigida por Hilarión Mamani, un ex cooperativista minero, y empezaron las consabidas acciones de mutuas expulsiones, etc. Pero lo que el MAS hasta ahora no ha podido lograr, ni con CONAMAQ ni con CIDOB (y sus múltiples organizaciones étnicas afiliadas), ha sido hacer desaparecer a la otra rama histórica.

Aquí la paro, sin entrar ya en otros actores que, sin estar del todo ausentes, tienen ahora menos protagonismo, como la COB (que fue durante décadas el gran paraguas para todos) o los sindicatos de maestros, y otros más presentes como los gremialistas y los transportistas.

\section{Autonomías}

La ingeniería constitucional de las tres principales formas de autonomía es compleja y no es todavía mucho lo que se ha logrado. Resumamos los rasgos, logros y tareas pendientes en esas tres formas principales: ${ }^{10}$

La autonomía municipal es la que mejor funciona, pues, según la Ley Marco de Autonomías y Descentralización (LMAD), del 2010, puede hacerlo incluso sin tener siquiera sus "cartas orgánicas". La razón es que en el fondo se ve solo como una profundización de lo que ya se había iniciado en 1994, durante el gobierno de Sánchez de Lozada, con la Ley de Participación Popular (LPP). Esta podría considerarse desde varias vertientes: desde el Estado, como una vía para mejorar la presencia del Estado a niveles locales; desde la coyuntura local oriental, como una medida para descolocar las pretensiones autonómicas departamentales sobre todo en Santa Cruz y Tarija; o también como el principio de un nuevo estilo de Estado, que es lo que ahora se busca.

10 Hay una cuarta forma, las autonomías regionales que, en los ajustes constitucionales realizados con la oposición a fines del 2008, quedó un poco en el limbo, reducida a arreglos prácticos sin consistencia propia, salvo tal vez como la instancia superior dentro de la autonomía IOC. 
De hecho gracias a ello los municipios o secciones municipales dentro de las provincias -que existían desde hace años- recién empezaron a tener recursos suficientes; antes los recursos estaban en manos de las Corporaciones Departamentales de Desarrollo, como la instancia local privilegiada para el desarrollo local.

En este sentido la Ley de Participación Popular resultó, en su momento, la más revolucionaria a este nivel, después de las reformas agrarias de los años 1950. Y quienes al comienzo la tildaban de "ley maldita del Banco Mundial" pronto la llamaron la "ley bendita". Lo que, en este contexto, ha hecho el MAS ha sido fortalecer esta instancia dándole nuevas competencias, incluida la de hacer leyes a ese nivel y otorgarle muchos más recursos. Por eso ya no es solamente descentralización sino autonomía.

En las autonomías departamentales, había dos situaciones distintas en y fuera de la Media Luna:

1. Los cuatro departamentos de la Media Luna, que ya reclamaban esta autonomía desde cuando el nivel central del Estado no quería oír hablar de ella, ya habían redactado sus estatutos (con grupos de trabajo bastante reducidos) y los aprobaron después en un cuestionado referendo que el Gobierno consideró ilegal, realizado antes de que se aprobara la nueva CPE 2009. Ahora, por tanto, necesita adecuar varios artículos, pero solo Pando ha culminado este ajuste y ya lo tiene vigente. Santa Cruz, que era antes la abanderada, no ha hecho todavía ningún intento sistemático de adecuación constitucional, en protesta por haber perdido alguna competencia (que para ellos debería ser autónoma al nivel departamental) sobre todo con relación a la titulación y redistribución de tierras, tema que la CPE reserva al nivel central del Estado. Los departamentos de Beni y Tarija están ya en etapas intermedias bastante avanzadas. Pero, de alguna forma, en diversas leyes de acople a la CPE 2009, se han incorporado elementos de aquel cuestionado referendo, por ejemplo, los necesarios para definir la composición de la Asamblea Legislativa Departamental. Esto quiere decir que el Gobierno ha acabado reconociendo "en grande" aquellos referendos y, por lo mismo, una vez reformulados los artículos anticonstitucionales, ya no exige para esos departamentos un nuevo referendo.

2. En cambio, en los cinco departamentos collas, esta temática se planteó solamente después de haberse aprobado la nueva CPE. La Paz, Oruro y Potosí ya han hecho su estatuto y también su control de constitucionalidad ante el Tribunal Constitucional Plurinacional (TCP); ahora les falta solo el 
referéndum final. Los departamentos de Chuquisaca y Cochabamba están algo más retrasados.

Las autonomías indígenas son de estilo diferente y siguen siendo las cenicientas. En rigor técnico, muchas de las autonomías municipales podrían ser indígenas por su alto porcentaje de población indígena. Pero únicamente 12 se lanzaron a esa aventura de dotarse de otra forma inédita de gobierno en 2009: a fines de ese año los 12 pioneros hicieron un referendo sobre si querían o no iniciar ese proceso; en 11 ganó el sí y siguieron la carrera. Cuatro años después tan solo tres han completado todos los requisitos salvo el referendo final aprobatorio del estatuto. Los demás están en fases anteriores y algunos simplemente se han estancado. Se suponía que al menos esos tres harían el referéndum pendiente antes de las elecciones subnacionales del 29 de marzo [2015], pues ya lo tenían solicitado ante el Tribunal Electoral desde la mitad del 2014; pero, a última hora, el Gobierno lo ha postergado para después, apelando a que el Tribunal Electoral ya está saturado de tareas en esas elecciones regulares, lo que también es cierto. Pero con ello se está creando una situación medio rara, porque quienes resulten electos a fines de marzo como alcaldes y concejales, deberán renunciar a ese cargo cuando meses después se realice el referendo sobre el estatuto autonómico IOC, si en él gana el "si", para dotarse de un gobierno según dicho estatuto. ¿Aceptarán dejar el sillón y sueldo recién logrado? ¿O habrá sido una trampa para postergar o deformar indefinidamente ese proceso ${ }^{11}$

${ }^{11} \mathrm{Al}$ menos en el caso del municipio de Charagua que por su tamaño (mayor a dos veces el departamento de Tarija aunque mucho menos poblado) es el más significativo para todo el pueblo guaraní. Esta situación puede exigir una solución salomónica ad hoc, porque el candidato a alcalde por el MAS y a la vez por las cuatro capitanías guaraní de la APG, don Belarmino Solano, fue el vicepresidente de la Asamblea Constituyente local que en los años anteriores ha estado elaborando el Estatuto ahora ya aprobado y avalado por el Tribunal Constitucional, y que solo espera la confirmación de la población con ese referendo final.

También ha recorrido todo el camino para transformarse en AIOC, la TCO/TIOC quechua de Raqaypampa en Cochabamba. Su estatuto ya había sido redactado en 2010, con apoyo del después senador del MAS Adolfo Mendoza, pasando a ser el primer ejemplo de AIOC por la vía TCO/TIOC (tierras comunitarias de origen, ahora llamadas ya territorios indígena originario campesinos), que en el país ya son más de 200 tanto en tierras bajas como en el altiplano). Por ser TCO/TIOC y no municipio, no necesita el referendo inicial ni los dos tercios de sus constituyentes, pero sí el control constitucional y el referendo final (CPE art. 275 y LMAD art. 54 III). Pero en lo que sí se necesita mucho cuidado en esa vía es de no cruzar límites de varios municipios, porque entonces necesitarían toda una ley de la Asamblea Legislativa. 
Nótese de paso la incoherencia entre los fáciles procedimientos para ser autonomía municipal y el largo proceso y costo para ser autonomía IOC, según la LMAD. Para que la primera entre en vigencia ni siquiera necesitan haber redactado su "carta orgánica" (equivalente a estatuto municipal) y, una vez redactada, basta que la aprueben dos tercios de los cinco concejales (o de los siete si el municipio es mayor).

En cambio, si -como en el caso de Charagua- se animan a transformarse en $\mathrm{AIOC}$, tienen que transitar por una serie de pasos previos, gastando mucho tiempo y dinero. Simplificando los pasos: a) ganar el referendo inicial para empezar el proceso; b) elegir a su asamblea constituyente local para que redacte el estatuto, con todas las negociaciones internas previas sobre su composición; c) una vez concluido este, aprobarlo por dos tercios de sus miembros; d) pasarlo al TCP para su control de constitucionalidad; e) organizar un nuevo referendo aprobatorio del estatuto. Implícitamente esa ley parece decirles: "Si les basta la autonomía municipal, no necesitan hacer nada y pueden aplicarla de inmediato. Pero si tienen la audacia de solicitar su transformación en $\mathrm{AIOC}$, ármense de valor, dinero, tiempo y paciencia".

El Consejo Nacional Autonómico, previsto en la LMAD de 2010, recién tuvo su primera sesión el pasado 11 de febrero de 2015. Está presidido por el Presidente e incluye a los ministros de la Presidencia, de Autonomías y Descentralización y de Desarrollo Sostenible; a los nueve gobernadores autonómicos departamentales; a cinco representantes de las autonomías municipales, a otros cinco de las autonomías indígenas y a uno de las regionales. En esa sesión inaugural Evo enfatizó la necesidad de lograr en esa especie de nueva instancia consultiva y de coordinación, una actitud de solidaridad hacia el bien común, mediante el aún pendiente "pacto fiscal" entre los que reciben más de lo que es indispensable y los que necesitan mucho más de lo que reciben; sugirió también que modificará la LMAD para incorporar al ministro de Economía y transformar las dos sesiones previstas actualmente por algo más permanente. Además, propuso los cuatro pilares sobre los que debiera asentarse ahora el desarrollo boliviano: hidrocarburos, minería, desarrollo agropecuario sostenible y servicios, explicitando en estos últimos también el turismo. Es demasiado pronto para saber si ello marca ya un nuevo estilo para el país o si se quedará simplemente en buenos deseos. 


\section{Epílogo: Otra cosa es con guitarra}

En Bolivia tenemos el dicho "otra cosa es con guitarra", entendiendo por "guitarra" el hecho de estar o no ejerciendo el poder. Cuando alguien o un grupo aún no está en el poder, tiene mucha más posibilidad de soñar y hacer dibujo libre, tomando en cuenta solamente las variables o factores que le interesan. En cambio, "con guitarra" tienen que tomarse en cuenta todos los factores y actores, sus intereses, con frecuencia en conflicto, y su respectiva correlación de fuerzas. No queda más remedio que ser realista. Muchos sueños se van relativizando y muchas veces hay que tomar al final soluciones intermedias, salomónicas, que mantengan el equilibrio, la "gobernabilidad" y la "gobernanza" entre todos.

Entra además en juego otro factor más perverso. Se dice que una vez estando en el poder, saltan a la vista las ambiciones ocultas de quienes están encaramados en ese "maravilloso instrumento" (como repetía don Víctor Paz Estenssoro), muchas veces ya no para implementar los sueños de país que se tenían antes, sino para usarlo según las propias conveniencias, mantenerse en él y seguir gozando de sus mieles y placeres. El poder corrompe y el poder absoluto corrompe absolutamente. Para evitarlo, el poder tiene que ser compartido, pluralista y hay que mantener claros cuáles eran los objetivos y la utopía por la que se quiso llegar a él, tomando en cuenta los intereses reales de cada actor.

En el caso boliviano, parecería que, desde el poder, ya no hay tanto interés para avanzar a fondo en algunos de los procesos "plurinacionales" señalados en la CPE. Siempre ha habido una tensión dialéctica entre la necesidad de tener un Estado fuerte y una apertura hacia lo plurinacional y diferente, con mayores niveles de participación, co-gestión y autonomía. El riesgo es que lo "pluri" entonces se vaya reduciendo a elementos secundarios y quizás simbólicos, dentro de un Estado relativamente unitario e igual, salvo en diferencias operativas menores.

Para mí, el gran desafío en la coyuntura actual es que Bolivia llegue a ser, con su CPE y su práctica, un modelo de país inter (lo.que.sea):

a) Dentro del país: ser inter-cultural e inter-étnico, inter-género, intergeneracional, urbano-rural quizás con doble residencia, de inter-regiones ecológicas con diversas posibilidades de desarrollo, inter-partidarios, interreligiosos, etc.

b) Con el resto de América Latina y más allá: inter-oceánico, abrirse a las relaciones inter-fronterizas, sobre todo en su triple frontera con Perú y Chile; 
abrirse a las migraciones en ambas direcciones; transformar esas fronteras en puente y no en muros infranqueables, como puente entre la Comunidad Andina y el Mercosur.

La condición para todo ello es mantener firmes las propias raíces y, a la vez, mostrar gran apertura a todos los otros diversos, pero de igual a igual. Esa convivencia interactiva será la base de su y nuestro buen (con)vivir felices entre nosotros, con la Madre Tierra y con nuestras diversas espiritualidades y cosmovisiones.

\section{Bibliografía citada}

ESTADO PLURINACIONAL DE BOLIVIA. 2007. Plan Nacional de Desarrollo: Bolivia digna, soberana, productiva y democrática para Vivir Bien. La Paz: Ministerio de Planificación del Desarrollo.

\section{(cc) $\mathrm{EY}$}

UILIS
New articles in this journal are licensed under a Creative Commons Attribution 4.0 United States License.

This journal is published by the University Library System of the University of Pittsburgh as part of its D-Scribe Digital Publishing Program, and is cosponsored by the University of Pittsburgh Press. 